\title{
La preuve juridique à l'épreuve du principe de précaution
}

\section{Alexandre Flückiger}

\section{(2) OpenEdition}

1 Journals

Édition électronique

URL : http://journals.openedition.org/ress/385

DOI : $10.4000 /$ ress.385

ISSN : 1663-4446

Éditeur

Librairie Droz

\section{Édition imprimée}

Date de publication : 10 décembre 2003

Pagination : 107-127

ISBN : 2-600-00913-2

ISSN : 0048-8046

\section{Référence électronique}

Alexandre Flückiger, «La preuve juridique à l'épreuve du principe de précaution », Revue européenne des sciences sociales [En ligne], XLI-128 | 2003, mis en ligne le 11 novembre 2009, consulté le 10 décembre 2020. URL : http://journals.openedition.org/ress/385; DOI : https://doi.org/10.4000/ress. 385

(c) Librairie Droz 


\section{LA PREUVE JURIDIQUE À L'ÉPREUVE DU PRINCIPE DE PRÉCAUTION}

SOMMAIRE: 1. L'incertitude juridique et l'incertitude scientifique. - 2. La preuve juridique en contexte d'incertitude scientifique. -3 . Les particularités des mesures prises en application du principe de précaution. -4 . Conclusion.

Le principe de précaution vise à adopter des mesures de prévention destinées à prévenir des dommages potentiellement graves et difficilement réversibles dont le lien de causalité n'est pas scientifiquement prouvé en l'état actuel des connaissances ${ }^{1}$. L'émergence de ce principe dans l'ordre juridique pose la question de la preuve: le système scientifique ayant échoué à administrer celle-ci, le droit y est invité. Le fardeau de la preuve serait-il donc renversé au profit des juristes? Le système probatoire juridique supportera-t-il l'épreuve d'un tel principe: peut-on légitimement prétendre prouver, en droit, l'improuvable en science?

On abordera ces questions en distinguant tout d'abord les particularités de l'incertitude juridique et scientifique en opposant l'une à l'autre (ch. 1). On examinera ensuite la preuve juridique dans le contexte spécifique de l'incertitude scientifique (ch. 2) pour évoquer les particularités des mesures prises sur le fondement du principe de précaution (ch. 3).

\section{L'INCERTITUDE JURIDIQUE ET L'INCERTITUDE SCIENTIFIQUE}

Paradigme probablement dominant de la vérité dans nos sociétés contemporaines industrialisées, la preuve scientifique représente vraisemblablement l'idéal de la vérité dans l'opinion commune. Mais la vérité ne s'accommode pas du singulier selon la formule de Paul Ricœur: même si «nous voulons que la vérité soit au singulier, l'esprit de vérité est de respecter la complexité» des «ordres de vérité : c'est l'aveu du pluriel» ${ }^{2}$. Parmi ces multiples vérités, l'une est particulièrement familière au juriste: la vérité judiciaire - qui ne saurait se confondre avec la vérité scientifique, même si l'époque n'est plus à la vérité judiciaire d'ordre divin matérialisée par un système de preuves irrationnelles et dangereuses telles que le duel judiciaire ou l'ordalie ${ }^{3}$.

Voir ci-dessous ch. 2.1.1.

Ricoeur 1955, p. 156 et 175 (cité in Kerchove 2000, p. 95).

Sur l'histoire de la preuve judiciaire, voir par exemple Lévy-Bruhl 1964. 
La première spécificité réside dans le fait qu'aucun jugement sur lequel se fonde la décision judiciaire, même celui qui établit simplement la réalité matérielle des faits, ne possède une nature purement constative ${ }^{4}$. L'autorité de la chose jugée ou décidée lui confère une nature en partie normative ou, plus exactement, performative en ce sens que son énonciation suffit à transformer immédiatement la réalités . Cette dimension de la décision judiciaire ne se prête pas à une évaluation en termes de vérité dans son acception scientifique, contrairement à l'aspect purement constatif des jugements à la base de cette décision. C'est cette dernière particularité qui justifie l'attribution d'une présomption irréfragable de vérité à la chose jugée (res iudicata pro veritate habetur). Il n'est dès lors pas justifié, selon van de Kerchove, «d'entretenir la fiction de la vérité scientifique absolue inhérente aux décisions judiciaires pour rendre compte du fait que les jugements constatifs sur lesquels elles se fondent bénéficient juridiquement d' une présomption irréfragable de vérité et qu'elles bénéficient elles-mêmes d'une autorité qui, dans les limites fixées par la loi, est considérée comme irrévocable.» ${ }^{6}$ Bruno Latour, dans une comparaison ethnographique créative entre juristes et scientifiques, après avoir affirmé que «si la chose jugée ne doit pas être prise "pour la vérité», ce n'est pas pour inaugurer quelque cynisme blasé, c'est qu' elle a bien mieux à faire que de mimer ou d'approximer le vrai: elle doit produire le juste, dire le droit, dans l'état actuel des textes» ${ }^{7}$, conclut qu' «il devient urgent de ne pas demander aux sciences de trancher, de ne pas exiger du droit qu' il dise vrai $»^{8}$.

En deuxième lieu, la vérité judiciaire s'écarte de la vérité scientifique dans la mesure où, tout d'abord, seule une partie des faits doit être retenue comme objet de preuve pour établir un droit (les faits dits «pertinents»). Qu'un piéton ait traversé la rue un quart d'heure plus tard qu'à son habitude et qu'il ait été renversé par une voiture sur un passage protégé n'est ainsi pas un fait pertinent, bien que l'accident n'eût pas eu lieu si le piéton avait été ponctuel'. La loi, ensuite, fixe des limites qui n'ont pas pour seul objectif de manifester la vérité. Toutes les preuves ne sont pas admissibles, car tous les moyens ne sont pas nécessairement bons pour les obtenir ${ }^{10}$. La découverte de la vérité n'est pas l'objectif exclusif du procès ${ }^{11}$ et ne doit certainement pas le devenir. Perelman le rappelle:

les techniques de la preuve et la vérité qu'elles doivent faire admettre [... doivent être] conciliables avec d'autres valeurs considérées, parfois, comme plus importantes ${ }^{12}$.

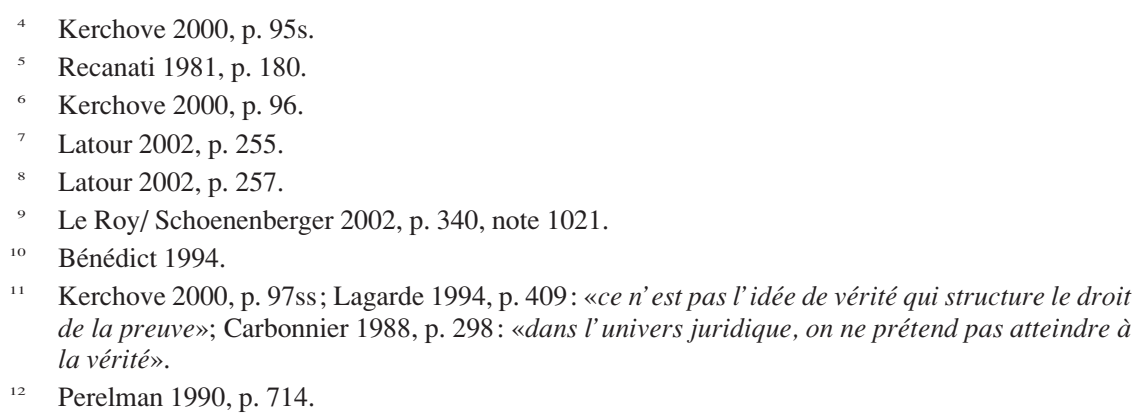


D'autres valeurs, que l'ordre juridique qualifie de plus importantes, doivent selon les circonstances l'emporter sur la recherche de la vérité. Le premier exemple est tiré des droits fondamentaux à l'instar de la liberté personnelle qui conduit à rejeter les moyens de preuve obtenus sous la torture ou par des traitements inhumains ou dégradants. L'affaire Magnus $G$. actuellement pendante devant le Landgericht de Francfort-sur-le-Main est à cet égard exemplaire ${ }^{13}$ : l'inspecteur Daschner a ordonné de durcir les interrogatoires jusqu'à l'utilisation de la violence si nécessaire pour arracher des aveux à un suspect. Il croyait qu'un enfant de onze ans, kidnappé puis étranglé, était encore en vie et courait un danger mortel aigu. En dix minutes, sous la menace de la torture, le suspect révèle où se trouve l'enfant enlevé. La police retrouve peu après le corps sans vie au fond d'un lac. L'inspecteur s'est dénoncé le même jour au juge. Une dose de torture contre la vie d'un enfant? L'ordre juridique constitutionnel et international la prohibe et un aveu obtenu dans de telles conditions doit être rejeté ${ }^{14}$. Si d'autres éléments de preuve admissibles et suffisamment convaincants ne peuvent faire la lumière sur les faits, toute la vérité ne pourra pas dans ce cas se manifester. Autour de la controverse engendrée par cette affaire (l'inspecteur Daschner est-il un héros ou un criminel inquisiteur?), on peut discerner une évolution - en droit pénal plus particulièrement - consistant à admettre plus largement des preuves obtenues au détriment du respect des droits fondamentaux ${ }^{15}$. Cette attitude est motivée pour des motifs sécuritaires afin d'avoir un maximum d'efficacité pour lutter contre les infractions normalement les plus graves. L'abandon partiel de ces valeurs n'est pourtant pas sans éveiller une certaine inquiétude. Dans ce débat, «la seule question à trancher, conclut van de Kerchove, est celle de savoir quels sont les objectifs dont, au regard des valeurs admises dans une société démocratique, la protection doit l'emporter, et dans quelles limites, sur le souci de manifester la vérité» ${ }^{16}$. En outre, même si le fait allégué n'est pas d'une certitude absolue, l'ordre juridique peut admettre le principe de l' «intime conviction » qui permet au juge de tenir un fait pour prouvé si celui-ci est persuadé de son existence sur la base des preuves apportées ${ }^{17}$. Dans ce système, les preuves sont appréciées librement si bien que même des indices concordants en faveur d'une hypothèse - ne constituant pas en eux-même de preuve - peuvent emporter valablement la conviction du juge ${ }^{18}$. Enfin, la vérité judiciaire peut résulter d'un pur raisonne-

13 http://www.landgericht.frankfurt-main.de

14 L'article 15 de la convention du 10 décembre 1984 contre la torture et autres peines ou traitements cruels, inhumains ou dégradants impose que «tout Etat partie veille à ce que toute déclaration dont il est établi qu' elle a été obtenue par la torture ne puisse être invoquée comme un élément de preuve dans une procédure, si ce n'est contre la personne accusée de torture pour établir qu' une déclaration a été faite.»

15 Kerchove 2000, p. 99. Voir également Cassani et al., à paraître. Une base légale vient d'être créée en Suisse pour l'utilisation des profils d'ADN et pour réglementer le recours à des agents infiltrés (loi fédérale sur l'utilisation de profils d'ADN dans le cadre d'une procédure pénale et sur l'identification de personnes inconnues ou disparues du 20 juin 2003, FF 2003 3981; loi fédérale sur l'investigation secrète du 20 juin 2003, FF 2003 4009).

16 Kerchove 2000, p. 98.

17 Le Roy/ Schoenenberger 2002, p. 358.

18 Piquerez 2001, ch. 1183. 
ment logique, volontairement abstrait de la réalité. La loi autorise ainsi à combler par le biais de présomptions les lacunes que les preuves laisseraient subsister (présomptions légales). La loi autorise ainsi soit à renverser la charge de la preuve (présomptions réfragables), soit à dispenser de prouver les faits qu'une partie allègue en interdisant la preuve du contraire (présomptions irréfragables). Dans le premier cas de figure, la présomption s'impose tant que la preuve du contraire n'est pas apportée, à l'exemple de la présomption d'appartenance au domaine public des eaux publiques, des régions impropres à la culture, des rochers, des éboulis, névés, glaciers et sources (art. 664 al. 2 du Code civil suisse). Ce type de règle peut paraître prima facie injuste, car il renverse le principe d'après lequel la preuve d'un fait incombe à la partie qui l'allègue (art. 8 du Code civil suisse) ${ }^{19}$. Il existe cependant des cas de figure où, les faits demeurant incertains, la loi a estimé qu'il serait plus légitime de faire supporter l'échec de la preuve à la partie qui ne les a pas allégués. Le juge ou l'administration ont le devoir de trancher un litige (interdiction du déni de justice) et ne peuvent pas se contenter de surseoir à leur décision jusqu'à ce que des preuves certaines soient trouvées. Anticipant les situations d'incertitude, la loi va donc aménager des exceptions chaque fois qu'elle estime qu'il est préférable que l'échec de la preuve profite à celui qui allègue un fait pour en déduire un droit. La loi opère ainsi un choix - politique - sur la partie à protéger en cas d'incertitude. En matière pénale, la présomption d'innocence (art. 32 al. $1^{\text {er }}$ Cst.) a pour effet qu'en cas d'incertitude le doute profite à l'accusé en ce sens qu'il ne peut être déclaré coupable tant que la présomption d'innocence n'est pas renversée (in dubio pro reo). Le but est ici de protéger l'accusé contre l'accusateur, représenté par l'appareil étatique qui dispose de moyens d'investigation considérables. Dans le second type de présomption (présomptions irréfragables), la preuve du contraire est simplement interdite au nom d'une valeur que la loi juge supérieure à la vérité, à l'exemple de la présomption de paternité qui devient irréfragable lorsque les délais pour contester une filiation sont dépassés (art. 256c du Code civil suisse). Ici, la loi évacue la question de la vérité au nom d'intérêts qu'elle juge supérieurs. Ce dernier exemple a pu aisément traverser les siècles, car la «fiction» ainsi entretenue était quasiment impossible à prouver avec certitude et cette preuve devenait toujours plus difficile administrer à la suite de l'écoulement du temps. On peut aujourd'hui se demander si les progrès technologiques ne vont pas peu à peu avoir raison de cette présomption au fur et à mesure que les tests de paternités disponibles en pharmacie se généraliseront ${ }^{20}$. La vérité scientifique l'emportera-t-elle bientôt sur la vérité judiciaire, au détriment de la paix des familles et de l'équilibre psychologique des «faux» enfants?

Troisième particularité différenciant vérité scientifique et judiciaire ${ }^{21}$, l'illusion déductiviste occultant la genèse profonde d'une conclusion juridique a pu faire croire que l'application d'un raisonnement syllogistique était suffisant pour

19 Ce principe est également valable en droit public (sur ses fondements en droit public, voir Moor 2002, p. 264).

20 Les premières réglementations sont en train de se préciser (projet de loi fédérale sur l'analyse génétique humaine [FF 2002, 6841]; recommandations du Préposé fédéral à la protection des données [du 22 janvier 2003 - Gentest.ch GmbH - et du 16 octobre 2002 - HBC Horizon Business Corporation $\mathrm{GmbH}]$ ).

${ }_{21}$ Sur une comparaison des raisonnements juridiques et scientifiques, voir Amselek 1987. 
empreindre le droit de rigueur «scientifique». Or, sans nier totalement l'importance d'une telle logique, le droit n'offre en réalité qu'une place limitée au raisonnement déductif comme les travaux de la nouvelle rhétorique notamment l'ont mis en évidence en soulignant l'importance de l'argumentation dont la force de conviction se révèle décisive pour provoquer l'adhésion du juge ou de l'autorité de décision ${ }^{22}$. Les faits - on vient de le montrer - doivent être construits et la qualification juridique, c'est-à-dire l'identification de la norme juridique étant donné les faits en cause, n'est pas un constat purement théorique ${ }^{23}$. Le programme de Perelman consiste précisément à étudier les moyens de «preuve» dont se sert le droit, non pas pour réfuter la théorie de la démonstration logique, mais pour la compléter par une théorie spécifique, celle de l'argumentation:

Les logiciens se doivent de compléter la théorie de la démonstration [...] par une théorie de l'argumentation. Nous chercherons à la construire en analysant les moyens de preuve dont se servent les sciences humaines, le droit et la philosophie ${ }^{24}$.

Henri Lévy-Bruhl propose dans ce sens de substituer le terme de conviction à celui de vérité, le premier lui semblant «plus approprié [...] à la preuve judiciaire» tout en précisant qu'il conviendrait également mieux aux autres preuves et «même à la preuve scientifique» ${ }^{25}$. Il en conclut que «prouver, c'est faire approuver $»^{26}$.

Les limites du raisonnement de logique formelle sont encore plus évidentes lorsque le droit est en présence de principes (par opposition aux règles au sens de Ronald Dworkin) ${ }^{27}$. La pesée des intérêts qui en résulte rend toute prévisibilité quant à la conclusion du jugement aléatoire. La jurisprudence relative au principe de précaution en offre une illustration éclatante ${ }^{28}$.

Inversement, on serait également victime d'une illusion si l'on pensait que les sciences exactes n'étaient basées que sur des preuves rigoureuses fondées sur un raisonnement purement déductif. La mise en évidence de la relativité de la vérité judiciaire ne devrait en effet pas contribuer à idéaliser la vérité scientifique. Karl Popper a montré que les théories scientifiques communément qualifiées de «vraies» ne pouvaient pas être prouvées; celles-ci n'ont simplement pas été réfutées avec succès pour l'instant ${ }^{29}$. Les travaux en philosophie et en sociologie des sciences ont enseigné que la méthode inductive de nature probabiliste (ou quasi inductive pour Karl Popper) côtoyait la démonstration déductive ${ }^{30}$ et que les

22 Perelman 1990, p. 696. Plus récemment: Solbach 2003; Ost/van de Kerchove 2002, p. 385ss; Fornel/ Passeron 2002. Sur les insuffisances de la subsomption et la proposition d'une logique alternative articulée autour de l'abduction, voir Papaux 2003.

23. Perelman 1992, p. 176: «il n' est pas possible [...] de rapprocher la rigueur du droit de celle des mathématiques.»

24 Perelman 1992, p. 13.

25 Lévy-Bruhl 1964, p. 21.

26 Lévy-Bruhl 1964, p. 22.

27 Sur le thème de la pesée des intérêts comme mode d'application des principes directeurs, voir Morand 1999, p. 83ss et 193s.

28 Voir ci-dessous, ch. 3.2.

29 Popper 1973, p. 270ss.

3о Popper 1973, p. 282ss. 
communautés scientifiques n'étaient pas spontanément et constamment consensuelles mais qu'elles fonctionnaient dans le débat et le conflit ${ }^{31}$. La vérité scientifique dans ces conditions ne peut être que relative. L'idéal de Descartes d'accéder par les ressources de la raison à l'univocité de la vérité se révèle de ce fait illusoire:

Toutes les fois que deux hommes portent sur la même chose un jugement contraire, il est certain que l'un des deux se trompe. Il y plus, aucun d'eux ne possède la vérité; car s'il en avait une vue claire et nette, il pourrait l'exposer à son adversaire de telle sorte qu'elle finirait par forcer sa conviction ${ }^{32}$.

\section{LA PREUVE JURIDIQUE EN CONTEXTE D'INCERTITUDE SCIENTIFIQUE}

Cette relativité de la certitude scientifique présente pourtant des degrés que le principe de précaution permet de mettre en évidence. La juridicisation de ce principe souligne l'autre relativité que nous venons d'examiner, celle de la certitude juridique. Le défi juridique, dans cette double mise en cause des dogmatismes, consiste à instaurer un mécanisme juste et politiquement acceptable permettant de prendre des mesures préventives dans un contexte de controverse scientifique: comment trancher équitablement lorsque des preuves indubitables n'ont pas pu être réunies? Le droit peut-il légitimement réussir là où la science a échoué?

Les exemples introductifs ont montré que le système juridique n'avait pas attendu l'apparition du principe de précaution pour être mis à l'épreuve de l'incertitude. Il n'est dès lors pas étonnant que les méthodes exposées à ce propos aient immédiatement été débattues pour être appliquées à ce nouveau principe. En particulier la question du degré de preuve (ch. 0) et celle de la répartition de la charge de l'administrer (ch. 0 ) que nous examinerons après avoir précisé la place de ce principe dans l'ordre juridique (ch. 0 ).

\subsection{Le principe de précaution dans l'ordre juridique}

\subsubsection{Définition}

Le principe de précaution vise à prendre des mesures préventives en présence d'un risque et d'un dommage potentiellement graves sans que ceux-ci ne puissent être fermement établis en l'état actuel des connaissances scientifiques ${ }^{33}$. Il se comprend le mieux en relation avec le principe de prévention dont il constitue le développement. Un examen de la distinction permettra de mieux saisir la particularité du premier.

Le principe de prévention, stricto sensu, vise à prévenir des dégâts dont le rapport de causalité avec le fait générateur du dommage est scientifiquement

\footnotetext{
Larrère 2002, p. 26.

32 Descartes, Règles pour la direction de l'esprit (réf. cit. in Perleman 1992, p. 2).

33 Pour une définition en droit suisse, voir Griffel 2001, p. 53ss; en France, voir Kourilsky/ Viney 1999, p. 10ss; en droit communautaire, voir Commission 2001; en droit international, voir Boisson de Chazournes 2002, p. 71ss.
} 
prouvé. Le principe de précaution est en revanche un jalon supplémentaire dans la réduction des risques, puisqu'il vise à adopter des mesures préventives lorsque ce lien ne l'est pas. Illustrons la distinction. En dépit de la relativité de la vérité scientifique, il existe des risques indubitablement certains dont le lien causal fait l'objet d'un consensus, à l'exemple des effets destructeurs de la foudre s'abattant sur un bâtiment dépourvu d'un paratonnerre. Le risque est certain; la seule incertitude réside en son occurrence dont la probabilité, toutefois, peut être évaluée, calculée et, partant, éventuellement assurée. La pose d'un parafoudre sera dans cette hypothèse une mesure de prévention. A l'inverse, il existe des risques dont la plausibilité d'un lien causal est discutée, à l'instar des effets des antennes de téléphonie mobile sur la santé des voisins. Les effets d'ordre biologique (modification du métabolisme de certaines cellules, troubles du sommeil, autres troubles du bienêtre ou effets éventuellement cancérigènes) ${ }^{34}$ ne sont actuellement pas prouvé scientifiquement. Différentes mesures destinées à diminuer le rayonnement des antennes, à les localiser de manière appropriée, voire carrément à les interdire dans certains endroits tels que dans les préaux d'école, constitueraient dans ce cas de figure des mesures de précaution. Dans le premier cas - pour reprendre la distinction, critiquée par Karl Popper ${ }^{35}$, entre probabilité d'événements et probabilité d'une hypothèse -, la probabilité de l'hypothèse scientifique à la base de la mesure préventive est certaine et celle d'événement est calculable alors que la probabilité d'une hypothèse fondant une mesure préventive prise sur la base du principe de précaution est en revanche incertaine et celle d'événement n'est pas quantifiable.

\subsubsection{L'émergence du principe de précaution dans l'ordre juridique}

Le principe de précaution s'insère peu à peu dans les ordres juridiques internes au sein desquels il se consolide graduellement. Bien qu'il existât déjà parfois discrètement par le détour du principe de prévention ou celui de proportionnalité ${ }^{36}$, c'est à la suite de sa concrétisation en droit international qu'il s'est vulgarisé. S'il visait à l'origine le domaine écologique, il tend à s'étendre à la protection de la santé en général. On illustrera brièvement cette évolution législative ${ }^{37}$ avec les exemples suivants.

En droit international, le principe de précaution s'affirme progressivement en tant que règle de droit positif ${ }^{38}$. Pour certains, il aurait déjà même acquis le statut d'une règle de droit coutumier ${ }^{39}$. En droit communautaire, il a été introduit à l'article $130 \mathrm{R}$ § 2 du Traité de l'Union européenne signé à Maastricht en 1992 (art. 174 du Traité d'Amsterdam). Il est repris à l'article III-124 du projet de Constitution européenne ${ }^{40}$. En droit suisse, il faut admettre que le principe de prévention

34 Ordonnance sur la protection contre le rayonnement non ionisant, Rapport explicatif, Berne, 16 février 1999, ch. 2.3 .

35 Popper 1973, p. 259ss.

36 Voir ci-dessous ch. 3.2

37 Sur la pratique jurisprudentielle, voir ci-dessous ch. 3.2.

38 Boisson de Chazournes 2002; Dupuy 2002.

39 Trouwborst 2002. Contra: Dupuy 2002, p. 95.

40 Convention européenne, Projet de Constitution, Volume II, Bruxelles, 12 juin 2003 (CONV 802/03). 
énoncé à l'article 74 al. 2 de la Constitution fédérale du 18 avril 1999 et à l'article $1^{\text {er }}$ al. 2 de la loi fédérale sur la protection de l'environnement du 7 octobre 1983 correspond au principe de précaution au sens de la distinction précédente ${ }^{41}$. Il est dès lors inexactement nommé en français principe de prévention, alors qu'il l'est correctement en allemand (Vorsorgeprinzip); différence linguistique que l'on ne retrouve plus à l'article 2 al. $1^{\text {er }}$ de la nouvelle loi fédérale sur l'application du génie génétique au domaine non humain du 21 mars $2003^{42}$. En droit français, l'expression a été employée pour la première fois dans la loi Barnier du 2 février 1995 relative au renforcement de la protection de l'environnement. Repris à l'article L 110-1 du Code de l'environnement et à l'article L 200-1 du Code rural, le principe de précaution sera peut-être bientôt de nature constitutionnelle ${ }^{43}$. Le projet de Charte de l'environnement remis par la commission Coppens le 8 avril 2003 contient deux versions du principe de précaution dont l'une, minimale, a déclenché un débat nourri ${ }^{44}$ sur lequel nous reviendrons ${ }^{45}$. Le projet de loi constitutionnelle relatif à la Charte de l'environnement a été adopté par le gouvernement en Conseil des ministres en été 2003.

\subsection{Quel degré de preuve pour l'incertain?}

L'action étatique est guidée par une série de principes garantissant le respect des valeurs d'un Etat de droit. Il convient dès lors de se demander si et à quelles conditions il est admissible de fonder une action en l'absence de preuves scientifiquement reconnues. Trois principes présentent ici un intérêt particulier ${ }^{46}$ : celui de légalité, de proportionnalité et d'interdiction de l'arbitraire ${ }^{47}$.

\subsubsection{Les principes de légalité, de proportionnalité et d'interdiction de l'arbitraire}

Le premier principe est celui de la légalité (art. 5 al. $1^{\text {er }}$ Cst.). Une loi doit tout d'abord habiliter l'Etat à prendre des mesures préventives. Si cette norme ne devait autoriser à agir qu'en cas de données scientifiques incontestables, l'Etat violerait la volonté démocratiquement exprimée par le législateur en prenant des mesures alors que règne une controverse scientifique majeure. L'émergence du principe de précaution dans les ordres juridiques nationaux et internationaux peut s'expliquer en partie dans ce contexte.

\footnotetext{
Alain Griffel 2001, p. 61; Petitpierre 2001, ch. 19. Contra: Epiney/ Scheyli 1998, p. 107.

42 FF 20032462.

43 Prieur 2003, p. 38ss.

44 Lepage 2003.

45 Voir ci-dessous ch. 3.2.

${ }^{46}$ Nous omettons d'examiner l'intérêt public notamment (art. 5 al. 2 Cst.) dans la mesure où la prévention de dommages graves ne relève pas de la poursuite d'intérêts particuliers purement privés.

47 En droit suisse, voir Griffel 2001,p. 117ss. En droit communautaire, sur les principes généraux «d'une bonne gestion des risques» examinés par la Commission des Communautés européennes lors du recours au principe de précaution, voir Commission 2000, p. 18ss (proportionnalité, nondiscrimination, cohérence, examen des avantages et charges, examen de l'évolution scientifique).
} 
Le second principe est celui de la proportionnalité (art. 5 al. 2 Cst.). Au cours de ce contrôle, l'autorité - législateur ou administration - vérifie en premier lieu si le moyen préventif choisi est propre à atteindre le but visé (critère de l'aptitude). Interprétée strictement, cette exigence n'autoriserait certainement pas l'Etat à prendre des mesures préventives en dehors d'un rapport de causalité dûment prouvé; règle que le principe de précaution permet précisément de tempérer. En droit suisse, le Tribunal fédéral, par le biais du principe de proportionnalité a appliqué il y a quelques années déjà le principe de précaution - sans le mentionner explicitement - en diminuant les exigences en matière de preuve dans des situations dans lesquels des facteurs d'incertitude sont inhérents, en l'occurrence l'interdiction du canoë sur des rivières dans lesquelles des espèces animales sont en voie de disparition:

\begin{abstract}
Es liegt in der Natur der Sache, dass das tatsächliche Ausmass solcher Risiken nicht im voraus sicher feststellbar ist; anderseits darf auch nicht verlangt werden, dass mögliche Risiken, solange sie nicht gutachtlich erhärtet und in ihrer Tragweite genau bestimmt sind, ignoriert werden und allfällige Verbote erst nach eingetretener nachgewiesener Schädigung der Natur Platz greifen dürfen. Dem Gesetzgeber steht in solchen Fällen bei der Abwägung der berührten Interessen und Bedürfnisse sowie der damit zusammenhängenden Risiken ein Spielraum offen ${ }^{48}$.
\end{abstract}

Pierre Moor a ainsi montré qu'en droit suisse le principe de la proportionnalité devait être interprété en gardant à l'esprit que la matière environnementale soulevait «autant de questions où la prudence semble être plus à sa place que la science ${ }^{49}$. Cette relativisation nécessaire du principe de proportionnalité a été formalisée très tôt dans la doctrine spécialisée ${ }^{50}$. Le droit communautaire a suivi une évolution semblable puisque c'est dans le cadre du contrôle de la proportionnalité que le principe de précaution a été implicitement appliqué avant sa consécration jurisprudentielle explicite ${ }^{51}$.

Troisièmement, l'action de l'Etat ne doit pas être arbitraire (art. 9 Cst.). L'autorité doit ainsi fonder sa décision sur des faits suffisamment établis. La réalité de tels faits doit dès lors être étayée par des preuves suffisantes ${ }^{52}$. Il est admissible de prévoir une diminution des exigences en matière de preuve si les circonstances l'exigent et de se contenter d'une simple vraisemblance. Par exemple en droit pénal, dans le domaine des faits justificatifs (devoir de fonction selon l'art. $32 \mathrm{du}$ Code pénal suisse ou légitime défense selon l'art. 33 du Code pénal suisse), une preuve stricte des faits excluant l'illégalité de l'acte litigieux n'est pas exigée; il suffit de les rendre vraisemblables et de déterminer s'ils sont plausibles ${ }^{53}$. Diminuer les exigences en matière de preuve sans pour autant décider hors de tout cadre rationnel minimal, tel est précisément l'enjeu pratique du principe de précaution. Un seuil minimal de scientificité est dès lors exigé.

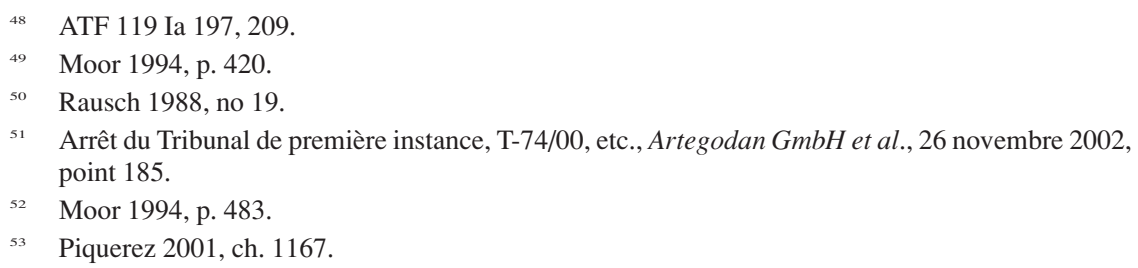




\subsubsection{Le seuil minimal de scientificité}

Afin que l'action préventive satisfasse aux critères de la proportionnalité et de la prohibition de l'arbitraire notamment, le principe de précaution ne saurait s'appliquer si la controverse scientifique n'a pas atteint un seuil minimal de consistance. Les risques hypothétiques reposant sur des intuitions purement spéculatives sans aucun fondement scientifique doivent être exclus du champ d'application du principe de précaution ${ }^{54}$. Il ne s'agit pas de céder, selon l'expression de Larrère, à «l' irrationalité d' une éthique de la peur, [...] ce qui aurait pour objet de bloquer aussi bien l'investigation scientifique que la délibération politique» ${ }^{55}$. La référence s'adresse à la théorie de l'heuristique de la peur d'Hans Jonas $^{56}$ - dont Bernard Sève a d'ailleurs mis en doute le caractère véritablement heuristique: on délibère pas pleinement sous la menace ${ }^{57}$.

Les mesures de précaution doivent donc, disions-nous, se baser sur un fondement scientifique présentant un minimum de consistance. Le Tribunal de première instance des Communautés européennes définit cette exigence par la formule suivante:

Le principe de précaution ne peut donc être appliqué que dans des situations de risque, notamment pour la santé humaine, qui, sans être fondé sur de simples hypothèses scientifiquement non vérifiées, n'a pas encore pu être pleinement démontré ${ }^{58}$.

Cette exigence va donner lieu en pratique à des débats délicats, car elle revient concrètement à définir un seuil de scientificité à partir duquel les avis minoritaires doivent effectivement être pris en considération. L'incertitude scientifique ne saurait être sans limite au regard du principe de précaution.

Là encore, le système juridique classique connaît déjà les outils appropriés : la preuve indiciaire, qui se contente de la vraisemblance ${ }^{59}$. Le plaideur devra fournir un minimum d'indices pour que naisse un doute suffisamment convaincant pour justifier les mesures de précaution ${ }^{60}$. Si ce mécanisme est conceptuellement clair, l'opposition des deux jurisprudences suivantes montre la difficulté concrète de définir ce seuil minimal. Dans l'affaire opposant l'Ecole polytechnique fédérale de Zurich à l'Office fédéral de l'environnement, des forêts et du paysage à propos du refus opposé par ce dernier d'autoriser un essai de culture de blé génétiquement modifié, le Département fédéral de l'environnement, des transports, de l'énergie et de la communication a jugé en septembre 2002 que l'administration «a interprété trop largement le principe de précaution rejetant la requête sur la base d' atteintes éventuelles purement hypothétiques» et qu' «elle n'a pas été en mesure

$54 \quad$ Sadeleer 1999, p. 176; Boisson de Chazournes 2002, p. 81.

s5 Larrère 2002, p. 23.

56 Jonas 1990, p. 49.

57 Sève 1993, p. 107ss.

s8 Tribunal de première instance des Communautés européennes, Pfizer Animal Health SA, 11 septembre 2002, point 146.

s9 Voir ci-dessus ch. 1 .

6о Sur un exemple d'utilisation d'indice dans le raisonnement juridique dans un cas d'application du principe de précaution, voir Tribunal de première instance des Communautés européennes, Pfizer Animal Health SA, 11 septembre 2002, points 244 et 297. 
d'exposer par quelles voies la résistance aux antibiotiques pouvait être transmise concrètement aux êtres humains et aux animaux» ${ }^{61}$. Dans l'affaire Pfizer Animal Health $S A$ c. Conseil où le risque potentiel concernait également le développement d'une résistance aux antibiotiques et son transfert à l'homme au travers de la chaîne alimentaire - mais en rapport avec l'utilisation d'un antibiotique dans l'alimentation animale -, le Tribunal de première instance des Communautés européennes a jugé en revanche à la même époque que les autorités pouvaient prendre une mesure préventive «même si, en raison de l'incertitude scientifique subsistante, la réalité et la gravité des risques pour la santé humaine liés à cette substance n'étaient pas encore pleinement démontrées»; les autorités n'étant «pas tenues, pour pouvoir agir d'une manière préventive, d'attendre que les effets adverses de l'utilisation de ce produit comme facteur de croissance se matérialisent ${ }^{62}$.

\subsection{A qui revient le fardeau de la preuve?}

\subsubsection{Le renversement du fardeau de la preuve : un problème mal posé}

Lorsque la diffusion d'un produit ou l'exploitation d'une installation sont soumises à la condition de leur innocuité, l'échec de la preuve du danger conduit à leur autorisation. Pareille éventualité est de moins en moins tolérée par l'opinion publique, s'il subsiste un doute, dans le domaine de la santé publique et de l'environnement notamment, là où les causalités sont complexes et où les dommages peuvent être graves et irréversibles. C'est dans cette conjoncture qu'une partie de la doctrine a réclamé un renversement du fardeau de la preuve en cas d'incertitude scientifique au bénéfice non plus du promoteur de l'activité litigieuse mais bien de la santé ou de l'environnement. Ces auteurs estiment qu'un tel renversement doit être déduit du principe de précaution, façonnant au passage un nouvel adage ( $\ll$ in dubio pro natura» $)^{63}$ calqué sur le modèle prévalant en droit pénal en cas de doute. Une controverse règne en doctrine pour savoir si le principe de précaution implique nécessairement un renversement du fardeau de la preuve ou non ${ }^{64}$. Le problème est le suivant: celui qui prend la mesure préventive n'a pas à prouver la causalité du dommage éventuel dans le système renversé, alors qu'il appartient à celui qui serait à l'origine du dommage potentiel de prouver l'innocuité de son action - preuve négative qu'il est difficile d'administrer dans un environnement scientifique précisément controversé; preuve diabolique donc pour certains (probatio diabolica) tant redoutée des juristes ${ }^{65}$. Cette opinion n'est exacte que si l'on exige dans ce cas un standard de preuve élevé, ce que certains paraissent

${ }^{61}$ Département fédéral de l'environnement, des transports, de l'énergie et de la communication, décision du 12 septembre 2002, ch. 2.3.5, p. 30.

62 Tribunal de première instance des Communautés européennes, Pfizer Animal Health SA c. Conseil et Alpharma c. Conseil, 11 septembre 2002, ch. 140 et 141.

63 Ost 1996, p. 192.

${ }_{64}$ La doctrine internationaliste dominante estime que le principe de précaution entraîne un renversement du fardeau de la preuve. Contra (avec réf. cit.): Cazala 2002, p. 168. Sur la nécessité de répartir le fardeau de la preuve plutôt que de le renverser, voir Belt/Gremmen 2002.

65 Dutheil de la Rochère 2002, p. 195; Cazala 2002, p. 171. 
déduire du renversement du fardeau de la preuve ${ }^{66}$. Qui peut pourtant prétendre avec une certitude absolue que tel produit n'aura pas telle incidence imprévue dans une génération ou deux? On est là au cœur de la problématique: ni la preuve incontestable de la nocivité, ni celle de son contraire ne peuvent être apportées dans le champ d'application du principe de précaution ${ }^{67}$. Dans un cas comme dans l'autre, tant que les connaissances scientifiques ne seront pas plus avancées et dans l'hypothèse où l'on ne se contente pas d'indices, cela signifie inévitablement une interdiction de l'activité si la charge de la preuve revient à son promoteur et, forcément, une autorisation si le fardeau revient à celui qui propose des mesures préventives.

Le problème est en réalité mal posé, car le fardeau revient déjà au promoteur de l'activité litigieuse lorsqu'un régime d'autorisation est instauré chaque fois qu'il est requérant d'une autorisation ou qu'il recourt contre une décision négative. L'analyse des techniques du renversement de la preuve le montrera ci-après. L'enjeu politique et juridique réside en réalité dans la combinaison entre la répartition de la charge de la preuve et le niveau de certitude exigé: pour les uns, la charge devrait reposer exclusivement sur le promoteur et le niveau de certitude scientifique être très élevé - ou, alors, être très bas si le fardeau devait reposer sur l'auteur des mesures préventives. Pour les autres, la situation inverse devrait prévaloir.

\subsubsection{Les techniques du renversement du fardeau de la preuve}

Techniquement, renverser le fardeau de la preuve revient à définir une présomption réfragable de risque en faveur d'installations déterminées ou de produits spécifiques que le promoteur peut tenter de lever en apportant simplement des indices en ce sens si un degré de certitude élevé n'est pas exigé. En cas d'échec de la preuve, si un doute devait subsister quant à l'innocuité du produit ou de l'installation, la présomption de dangerosité subsistera. En revanche, s'il doit en prouver l'innocuité absolue, pareille exigence constituerait en l'état actuel des connaissances scientifiques une preuve «diabolique» impossible à apporter puisqu'on se trouve par hypothèse dans le champ d'application du principe de précaution, rendant de fait la présomption irréfragable ${ }^{68}$.

66 Dutheil de la Rochère 2002, p. 195; Cazala 2002 paraît l'admettre implicitement en opposant la théorie du recours à la preuve prima facie - qu'il préconise - à celle du renversement de la preuve (voir p. 176).

${ }_{67}$ Dans le même sens, voir Sadeleer 1999, p. 193s.

68 Tel est précisément le problème que pose l'initiative populaire fédérale intitulée «Moratoire sur les antennes de téléphonie mobile», actuellement au stade de la récolte des signatures, dans la mesure où celle-ci propose d'ancrer dans la Constitution fédérale l'interdiction de construire de telles installations «tant que l'innocuité des rayons non ionisants pulsés et des champs magnétiques ou électromagnétiques pulsés, compte tenu de leurs effets non thermiques également, n'aura pas été démontrée.» Cette condition présume de manière irréfragable dans les faits la dangerosité des rayonnements visés puisqu'en l'état actuel des connaissances scientifiques, il est exclu d'en «démontrer» l'innocuité. L'initiative prévoit que le législateur sera compétent pour lever la présomption, et non un juge («La présomption de nocivité [...] ne pourra être levée que par la loi») (FF 2002 1950). 
En pratique, le droit positif introduit ce type de présomption chaque fois qu'il instaure un régime d'autorisation préalable subordonné à la condition de rendre vraisemblable l'absence de danger sur la santé ou l'environnement ${ }^{69}$. La distribution du produit ou l'exploitation de l'installation sont interdites tant qu'une autorisation n'est pas délivrée. La preuve de la compatibilité environnementale revient alors au requérant de l'autorisation en vertu des règles ordinaires sur la répartition du fardeau probatoire. En cas d'échec, l'autorisation sera refusée. Tel est le cas par exemple de l'interdiction de commercialiser des organismes génétiquement modifiés lorsque ceux-ci peuvent constituer une menace pour l'environnement ou pour l'homme avec la charge imposée au producteur d'effectuer à cette fin un contrôle autonome (art. 29b LPE). En revanche, en cas de recours contre la décision d'autorisation, la charge de la preuve revient au recourant. C'est-à-dire, si l'autorisation a été préalablement refusée, au requérant qui supporte derechef le fardeau. $\mathrm{Si}$ au contraire le conflit porte sur une autorisation précédemment accordée, le requérant tirera profit de l'échec de la preuve alors qu'il incombera au recourant (organisation écologiste, voisin, etc.) de démontrer que l'autorisation comporte des risques. C'est cette seconde configuration qui est certainement à l'origine du débat politique autour du renversement de la charge de la preuve. Dans ce cas, le fardeau, bien que non renversé, est pourtant allégé dans une certaine mesure par une particularité de la procédure administrative: celle d'être dominée par la maxime inquisitoriale contraignant l'administration à établir ellemême les faits pertinents sans attendre que l'administré fournisse sponte sua les preuves adéquates ${ }^{70}$.

Le législateur peut faire un pas de plus et introduire une présomption irréfragable de la nocivité d'un produit en l'interdisant purement et simplement sans instaurer un régime d'autorisation permettant d'administrer la preuve du contraire. Ce régime peut être assoupli en ce sens que le législateur peut prévoir un régime transitoire ou des dérogations exceptionnelles, à l'instar de l'interdiction des chlorofluorocarbones (annexe 3.4 de l'ordonnance sur les substances dangereuses pour l'environnement du 9 juin $1986^{71}$ ). Lorsque la durée d'une telle interdiction est limitée dans le temps, elle est généralement qualifiée de moratoire. Seule l'autorité l'ayant décrété pourra autoriser ultérieurement le procédé en cas d'évolution des connaissances ou de modification des pouvoirs de force en annulant la présomption qu'elle avait adoptée (sous réserve de délégation). Le Parlement fédéral vient ainsi de refuser à deux reprises l'instauration d'un moratoire pour l'utilisation dans l'agriculture des organismes génétiquement modifiés $^{72}$. Une initiative populaire fédérale «Pour des aliments produits sans manipulations génétiques», actuellement au stade de la récolte des signatures,

En droit communautaire, voir Commission 2000, p. 22.

7o Moor 2002, p. 259.

71 RS 814.013.

72 Le Conseil national a décidé à une courte majorité (83 voix contre 78) en mai 2003 l'instauration jusqu'en 2009 d'un moratoire interdisant l'utilisation dans l'agriculture des organismes génétiquement modifiés (BO CN 2003 654) alors que le Parlement avait renoncé précédemment à prévoir un tel moratoire dans la loi sur le génie génétique du 21 mars 2003 (FF 2003 2462). Le Conseil des Etats a pour sa part refusé ce moratoire par 29 voix contre 6. Le Conseil national est alors revenu sur son refus initial (session du 12 juin 2003). 
propose un tel moratoire de cinq ans dès son adoption par le constituant ${ }^{73}$. Inversement, le législateur peut prévoir un délai transitoire autorisant l'utilisation de produits problématiques jusqu'à une certaine date ${ }^{74}$.

\section{LES PARTICULARITÉS \\ DES MESURES PRISES EN APPLICATION DU PRINCIPE DE PRÉCAUTION}

Lorsque les thèses scientifiques minoritaires ont convaincu les autorités de leur vraisemblance et emporté leur conviction en dépit des incertitudes scientifiques, la question du choix des mesures peut être posée. Ce choix ne relève pas d'une logique binaire (autorisé ou interdit) mais révèle une palette différenciée de mesures fondées sur le principe de précaution.

\subsection{L'obligation d'évaluer: une dynamisation de la décision juridique}

Le droit a développé différents mécanismes pour décider en situation d'incertitude à l'instar des normes finalisées, des actes de planification, des études d'impact, des mécanismes d'évaluation, etc., caractéristiques de la néo-modernité juridique selon Charles-Albert Morand ${ }^{75}$. On se concentrera dans ce cadre sur l'instrument de l'évaluation auquel il a été très tôt recouru en application du principe de précaution, car il permet d'introduire une dynamique dans le processus décisionnel. La décision n'est plus considérée comme un événement définitif, arbitrant irrévocablement le conflit, mais un jalon parmi d'autres dans un processus évolutif dont le sens résulte de l'ensemble. L'incertitude scientifique n'est ainsi pas «miraculeusement » transmuée en pseudo-certitude par sa juridicisation, mais se reflète dans un type de décision plus réaliste, empreints d'une dynamique de révision propre - une décision provisoire, «incertaine», doublée d'obligations positives d'évaluation, de suivi et de correction si nécessaire, voire d'exécution de programmes de recherche scientifique. Pas plus que les alchimistes ne se sont révélés capables de transmuer les métaux en or, les juristes ne sont aptes à faire oublier l'incertitude des faits par le tranchant, illusoire, de la vérité judiciaire.

La Cour constitutionnelle fédérale allemande a montré la voie à suivre dès 1978 à propos de l'autorisation d'exploiter un surgénérateur à Kalkar en Rhénanie-du-Nord-Westphalie près de la frontière hollandaise. La Cour a imposé

73 FF 2003 1046. L'initiative populaire fédérale intitulée «Moratoire sur les antennes de téléphonie mobile » mentionnée précédemment (réf. cit. in note de bas de page no 68) n'est en réalité pas un moratoire, car elle propose d'ancrer dans la Constitution fédérale l'interdiction de la construction de telles installations «tant que l'innocuité des rayons non ionisants pulsés et des champs magnétiques ou électromagnétiques pulsés, compte tenu de leurs effets non thermiques également, n'aura pas été démontrée» sans prévoir de délai.

74 L'article 37 de la loi sur le génie génétique précitée (voir note de bas de page no 72) prévoit ainsi que «l' utilisation, dans le cadre de disséminations expérimentales, de gènes qui induisent une résistance aux antibiotiques utilisés en médecine humaine ou vétérinaire est autorisée jusqu'au 31 décembre 2008.»

$75 \quad$ Morand 1999. 
au législateur, en cas d'incertitude, de réunir et d'exploiter systématiquement les données nécessaires à l'évaluation des effets produits par la loi et de corriger celle-ci en fonction de cette évaluation ${ }^{76}$ :

\begin{abstract}
En l'absence de situations concrètes passées, l'évaluation [de la probabilité de la survenance de dommages futurs dus à l'exploitation d'une installation nucléaire] devra se baser sur des simulations. Dans la mesure où il n'existerait dans ce domaine aucune certitude mais que des approximations, tout nouvel événement ainsi que tout nouveau développement des connaissances devront être pris en compte au fur et à mesure de leur survenance ${ }^{77}$.
\end{abstract}

Le Tribunal fédéral suisse a repris ce concept - sans se référer au droit allemand - pour décider de la conformité au droit fédéral de l'ordonnance du Conseil fédéral sur la protection contre le rayonnement non ionisant du 23 décembre $1999^{78}$ en imposant au gouvernement une obligation d'observation, d'évaluation et de correction de cette ordonnance; obligation qui peut également se baser, même si le Tribunal fédéral ne l'a pas fait explicitement, sur les articles 170 Cst. et $44 \mathrm{al} .1^{\text {er }}$ de la loi fédérale sur la protection de l'environnement obligeant d'évaluer l'efficacité des mesures prises par la Confédération ${ }^{79}$. Le Tribunal fédéral a jugé en l'espèce que le Conseil fédéral devra dans la mesure du possible procéder à un nouvel examen des valeurs limites fixées dans cette ordonnance lorsque de nouvelles connaissances scientifiques objectives et fiables sur les effets biologiques des rayonnements non ionisants seront disponibles et a débouté les recourants au motif que les expertises fournies ne démontraient pas selon lui l'existence de telles connaissances. Le Conseil fédéral dispose à cet égard d'une grande liberté d'appréciation selon le Tribunal fédéral ${ }^{80}$.

La jurisprudence précédente peut être discutée sous l'angle du principe de précaution $^{81}$, car les valeurs limites d'immission pour le rayonnement électromagnétique non ionisant correspondent aux valeurs préconisées dans les recommandations de la Commission internationale de la protection contre le rayonnement non ionisant; normes qui ne prennent pourtant en compte que les effets scientifiquement prouvés en omettant les effets biologiques, non prouvés. Le rapport explicatif relatif au projet d'ordonnance sur la protection contre le rayonnement non ionisant reconnaît pourtant lui-même cette insuffisance, précisant clairement que la protection contre les atteintes incommodantes n'est pas complètement garantie par cette ordonnance même lorsque les valeurs limites d'immission sont respectées, car ces valeurs ont été fixées sans tenir compte des effets non prouvables scientifiquement ${ }^{82}$. En attendant, le jugement satisfera tous les adeptes de

\footnotetext{
BverfGE 49, 89; Morand 1999, p. 112s.

77 BverfGE 49, 89, 143 (traduit in Sadeleer 1999, p. 154).

78 RS 814.710.

79 Voir également les art. 27, 44 al. $1^{\text {er }}$ let. e et f et 54 al. 4 de la loi sur le Parlement (LPar) (FF 2002 7577) et l'art. 10 du projet d'ordonnance de l'Assemblée fédérale portant application de la loi sur le Parlement et relative à l'administration du Parlement (OLPA) (FF 2003 4583).

so $\quad$ ATF 126 II 399 c. 3a; ATF du 8 avril 2002 (DEP 2002 427).

s1 Egalement critique à l'encontre de cette jurisprudence: Griffel 2001, p. 77, note 132.

82 Ordonnance sur la protection contre le rayonnement non ionisant, Rapport explicatif du 16 février 1999, ch. 3.2. Le Tribunal fédéral a consenti à ce mode de procéder au motif qu'il est possible de
} 
la téléphonie mobile qui se plaignent des coupures de communication dues aux zones d'ombre résiduelles au détriment en revanche des voisins des antennes qui suspectent des atteintes à leur santé.

\subsection{Vers un double seuil de scientificité: mesures matérielles et mesures procédurales}

Le débat précédent montre - implicitement du moins - qu'il existe en pratique, entre le seuil minimal de scientificité déclenchant le principe de précaution et celui de la certitude scientifique entraînant celui de prévention, un niveau qualitatif intermédiaire dans l'incertitude juridique en dessous duquel l'application du principe a exclusivement des conséquences d'ordre procédural (obligation d'évaluation périodique comme en l'espèce, voire de développer un programme de recherche tout en conservant le statu quo quant à l'exercice de l'activité litigieuse) et en dessus duquel l'application a des conséquences matérielles d'autant plus consistantes qu'approchant le niveau de certitude supérieur (limitation de l'activité dans le temps, à l'exemple des moratoires ou des mesures provisionnelles, ou dans l'espace, à l'exemple de normes protectrices accrues autour d'endroits sensibles; fixation de conditions spécifiques à l'exercice de l'activité en cause; interdiction pure et simple). Ce seuil de scientificité intermédiaire - que l'on pourrait qualifier de matériel par opposition au seuil minimal que l'on pourrait appeler formel - est justifié dans la mesure où il résulte de l'application combinée du principe de précaution avec celui de proportionnalité que nous avons déjà rencontré plus haut. La notion de seuil est bien sûr réductrice dans une telle perspective - l'image de l'échelle graduée conviendrait certainement mieux - mais elle permet d'appréhender plus finement l'état de la controverse autour de l'application du principe de précaution: pour les contempteurs de ce dernier, ce niveau devrait être la ligne rouge à ne jamais franchir en cas d'incertitude scientifique alors que les partisans y voient le repère minimal en dessus duquel le principe déploie véritablement son efficacité. La question que les seconds pourraient soulever est celle de savoir si les mesures formelles constituent de véritables mesures de précaution ou si seules les mesures matérielles méritent cette qualification. Assurer une veille technologique dans un domaine controversé, exécuter des programmes de recherche pointus sont sans doute des mesures de précaution, mais de très faible degré. Ne pas les exiger montrerait a contrario une absence totale de prudence. En tout état de cause, de tels actes forment indubitablement des mesures prises en application $d u$ principe de précaution pour qui leur dénierait l'étiquette de mesures de précaution.

Ce problème est exactement au centre de la polémique lancée en France par Corinne Lepage critiquant la double version du principe de précaution dans le projet de Charte de l'environnement élaboré par la commission Coppens, l'une érigeant la précaution en principe (variante 2) et l'autre non (variante 1):

Quand un risque de dommage à l'environnement, grave et difficilement réversible, a été identifié, sans qu'il puisse être établi avec certitude en l'état des connaissances scienti-

tenir compte de tels effets dans le cadre de la limitation préventive des émissions au sens de l'article 11 al. 2 LPE (ATF 126 II 399, 406 confirmé in ATF 128 I 59). 
fiques, les autorités publiques mettent en œuvre, par précaution, des procédures d'évaluation et prennent les mesures appropriées. (ch. 12, variante 1)

La préservation et la mise en valeur de l'environnement reposent sur [...] le principe de précaution selon lequel quand un risque de dommage grave ou irréversible à l'environnement ou à la santé a été identifié, sans qu'il puisse être établi avec certitude en l'état des connaissances scientifiques, l'autorité publique met en œuvre un programme de recherches et prend les mesures provisoires et proportionnées propres à y parer (ch. 12, variante 2).

L'argumentation invoquée pour éviter d'ériger la précaution en principe met précisément l'accent sur les conséquences essentiellement procédurales que ce principe devrait revêtir dans l'hypothèse où le seuil d'incertitude intermédiaire - le seuil qualifié de matériel - ne serait pas atteint:

\begin{abstract}
L'expression courante «principe de précaution» n'est pas retenue [dans la variante 1], pour ne pas donner à cette disposition la portée d'un principe matériel, alors que l'exigence d'une démarche de précaution implique en premier lieu la mise en œuvre de procédures de recherche et d'évaluation et, s'il y a lieu, dans l'attente de leurs résultats, des mesures adaptées ${ }^{83}$.
\end{abstract}

Les partisans de cet avis sont heurtés à l'idée d'officialiser la logique argumentative de la décision juridico-politique au détriment de la traditionnelle logique scientifique classique, seule digne d'être qualifiée d'objective à leurs yeux. Ils déplorent en outre que le principe de précaution, qui est l'expression même de la prudence, aille toujours dans le sens de la prudence... Ils désapprouvent en réalité l'existence même du principe de précaution.

\begin{abstract}
L'expression «principe de précaution » n'est pas souhaitable parce qu'elle est particulièrement ambiguë. Dans sa dimension substantive, le principe de précaution est un principe de décision. Plus exactement, il invite en situation d'incertitude, où des arguments en conflit ne peuvent pas être tranchés objectivement, à privilégier certains arguments contre d'autres alors même qu'il n'est pas possible de les départager au niveau scientifique. Cela va toujours dans le sens d'une interdiction, d'une restriction, d'une prudence, jamais dans celui de la prise d'un risque ${ }^{84}$.
\end{abstract}

La jurisprudence et la pratique administrative ont pourtant déjà reconnu un seuil minimal matériel de scientificité dans l'application du principe de précaution. En France par exemple pour justifier que le ministre de l'agriculture soit enjoint de réexaminer son refus de retirer du marché l'insecticide «Gaucho» utilisé pour le traitement des semences de maïs et de betteraves au motif de n'avoir pas suffisamment examiné les risques que celui-ci présentait pour les abeilles bien que rien ne prouvât de façon indubitable la responsabilité de l'insecticide litigieux ${ }^{85}$, pour suspendre une autorisation de mise en culture de maïs transgénique $^{86}$ ou pour empêcher le stockage de farines de viande et d'os à bas risque dans le Port du Rhin à Strasbourg alors que ces aliments provenaient de matériaux

\footnotetext{
83 Coppens 2003, p. 39.

${ }^{84}$ Coppens 2003, p. 39.

85 Conseil d'Etat, Union nationale de l'apiculture française, no 233876, 9 octobre 2002.

s6 Conseil d'Etat, Association Greenpeace France et al., no 194348 etc., 11 décembre 1998.
} 
qui ne présentent pas de risques sérieux d'être contaminés ${ }^{87}$. En droit communautaire également ${ }^{88}$, la Cour de justice a implicitement appliqué le principe de précaution pour justifier l'interdiction de filets maillants dérivants de plus de 2,5 $\mathrm{km}$ même en l'absence d'avis scientifiques ou à la lumière d'avis non concluants $^{89}$. Elle a également admis l'interdiction d'exporter tout bovin de Grande-Bretagne sans attendre que la réalité du risque de transmission de l'encéphalopathie spongiforme bovine à l'être humain soit clairement démontré ${ }^{90}$. Le Tribunal de première instance, dans un arrêt que nous avons mentionné plus haut, a pour sa part confirmé le retrait de l'autorisation de certains antibiotiques dans l'alimentation animale même si le transfert de résistance des antibiotiques à l'homme est encore sujet à caution ${ }^{91}$. En Suisse, outre la jurisprudence du Tribunal fédéral justifiant l'interdiction du canoë en rivière précité ${ }^{92}$, l'Office fédéral de la protection de l'environnement, des forêts et du paysage a refusé l'autorisation de cultiver en plein air à Oftringen un champ de maïs transgénique résistant aux herbicides en raison du risque de propagation du pollen de maïs transgénique sur des champs de maïs traditionnels. L'office s'est basé sur le principe de précaution (appelé, rappelons-le, principe de prévention dans le texte français) ${ }^{93}$ exigeant que les incertitudes liées aux effets à long terme des atteintes à l'environnement soient compensées par une marge de sécurité ${ }^{94}$. Dans une autre affaire, le même office a refusé sur le même fondement à la Station fédérale de recherches en production végétale de Changins l'autorisation de cultiver en plein champ des pommes de terre transgéniques dont la résistance au mildiou était testée en raison du risque de transfert de résistance aux antibiotiques ${ }^{95}$.

Ces exemples - qui, afin de brosser un tableau objectif, devraient être mis en relation avec les affaires dans lesquelles l'application du principe n'a pas été reconnue ou dans lesquelles le seuil minimal de scientificité matérielle n'a pas été admis - ne remettent toutefois pas en cause la particularité des mesures prises en

Tribunal administratif de Strasbourg, Association de défense des intérêts des quartiers centre-est de Strasbourg et al., no 01-00951, in Environnement : actualité du droit public, privé et pénal de l'environnement, no 4, avril 2002, ch. 58, p. 19s.

88 La liste de la jurisprudence complète reconnaissant le principe de précaution est énumérée par le Tribunal de première instance dans son arrêt du 11 septembre 2002 (T-13/99, Pfizer Animal Healh, no 115). Voir également Dutheil de la Rochère, 2002.

89 C-405/92, Mondiet, Rec. p. I-6133, 24 novembre 1993.

90 C-157/96, National Farmers' Union, Rec. p. I-2211, 5 mai 1998; C-180/96, Royaume-Uni, Rec. p. I-2265, 5 mai 1998.

91 T-13/99, Pfizer Animal Healh, 11 septembre 2002.

92 Voir ci-dessus ch. 2.2.1.

93 Voir ci-dessus ch. 2.1.2.

${ }_{94}$ Décision B98001 du 16 avril 1999 (FF 1999 3039) entrée en force (DEP 1999 746). Sur cette marge de sécurité résultant des incertitudes scientifiques, voir également Rausch 1988, no 19 et Griffel 2001, p. 60.

95 Décision B98002 du 16 avril 1999, FF 19992778 (DEP 1999 752). Cette décision a fait l'objet d'un recours qui a été rejeté sur la forme (défaut de qualité pour recourir) par le Département fédéral de l'environnement, des transports, de l'énergie et de la communication (décision 519.2/25 du 19 septembre 2000, DEP 2000, 728). L'office a également refusé un essai de culture de blé génétiquement modifié mais a été désavoué par l'instance de recours (voir ci-dessus ch. 2.2.1. i.f.). 
application du principe de précaution: c'est-à-dire leur nature dynamique, évolutive et provisoire. Empreintes de l'incertitude auxquelles elles sont contraintes de se référer, les décisions juridiques deviennent elles-mêmes en quelque sorte incertaines, phénomène renforcé par la nature même des principes dont le caractère indéterminé et la vocation à être appliqués par une pesée d'intérêts plutôt que par une déduction syllogistique rend tout pronostic sur la décision incertain ${ }^{96}$. Le jugement, quelle que soit son issue, ne peut être que formellement définitif. La présomption de vérité qui en découle n'est que provisoirement irréfragable, car le jugement ou la décision ne règlent dans le domaine de la précaution qu'une situation momentanée, susceptible d'être remise en question lors de la découverte d'un nouvel indice. Le processus normatif devient continu.

\section{CONCLUSION}

L'émergence du principe de précaution révèle une double relativité, celle de la preuve scientifique et celle de la preuve juridique. Elle désempare ceux qui attendent de la science une réponse certaine à tous les problèmes de société et désarçonne ceux qui auraient pu penser que la logique déductive du raisonnement juridique classique aurait pu pallier les limites de la méthode scientifique dans un contexte d'incertitude. La preuve juridique - mise à l'épreuve du principe de précaution - révèle à ceux qui l'auraient méconnu le caractère relatif de la vérité judiciaire. Le principe de précaution ne renvoie cependant pas le droit contemporain à l'époque pré-cartésienne des preuves judiciaires irrationnelles. L'incertitude scientifique ne saurait être totale afin de ne pas céder trop prestement à la panique devant toute nouvelle crainte trop rapidement diffusée. Le droit ne la reconnaît qu'à condition qu'un minimum d'indices confère aux thèses minoritaires une certaine vraisemblance. Un seuil minimal de scientificité est dès lors exigé afin d'exclure du champ d'application les discussions purement spéculatives. Combinant ce principe à celui de la proportionnalité, le système juridique permet en outre de nuancer l'(in)certitude scientifique en conférant aux décisions prises sur son fondement un caractère incertain - ou, plus précisément, provisoirement certain - reflétant les incertitudes qu'elle n'a pas dissipées.

L'émergence du principe de précaution dans la sphère politico-juridique correspond au final à un «retour en force de la décision politique», car «le décideur ne pourra plus trouver refuge derrière le paravent des pseudo-certitudes scientifiques que lui offre son expert» selon l'analyse de Sadeleer ${ }^{97}$. C'est un premier pas dans le sens de Bruno Latour, pour qui il faudrait, de manière générale, «retirer aux sciences le pouvoir de dire le dernier mot qu' on leur avait confié par erreur ou par lâcheté» ${ }^{98}$. Cette conclusion offre une piste de réponse à la ques-

96 Morand 1999, p. 83ss et 193s. Pour une illustration de la jurisprudence du Tribunal fédéral relative à l'application du principe de prévention dans le cadre des pesées des intérêts, voir réf. cit. in Griffel 2001, p. 127s.

97 Sadeleer 1999, p. 196. Dans le même sens, voir Larrère 2002, p. 21; Callon/ Lascoumes/ Barthe 2001.

$98 \quad$ Latour 2002, p. 254. 
tion finale posée par Pierre Livet et Henri Volken dans leur texte préparatoire au présent colloque ${ }^{99}$ : l'émergence progressive du principe de précaution montre qu'il est peut-être en passe d'être «possible et politiquement praticable» de concevoir la décision politique - et juridique par extension - «comme non détachable des incertitudes qu'elle n'a en fait pas levées».

\section{Université de Genève}

\section{BIBLIOGRAPHIE}

Amselek, Paul, Théorie du droit et science, Paris, 1994

Bénédict, Jérôme, Le sort des preuves illégales dans le procès pénal, Lausanne, 1994

Belt (van den), Henk/ Gremmen, Bart, «Between precautionary principle and „sound science“: distributing the burdens of proof», Journal of Agricultural and Environnmentlal Ethics, 15, p. 103ss

Boisson de Chazournes, Laurence, «Le principe de précaution: nature, contenu et limite», in Leben, Charles/Verhoeven, Joe (dir.), Le principe de précaution: aspects de droit international et communautaire, Paris, 2002, p. 65ss

Callon, Michel/ Lascoumes, Pierre/ Barthe, Yannick, Agir dans un monde incertain: éssai sur la démocratie technique, Paris, 2001

Carbonnier, Jean, Droit civil : introduction, $17^{\circ}$ éd., Paris, 1988

Cassani Ursula et al., «Plus de sécurité - moins de liberté: les techniques d'investigation et de preuve en question», Coire et Zurich, à paraître

Cazala, Julien, Principe de précaution et procédure devant le juge international, in Leben, Charles/Verhoeven, Joe (dir.), Le principe de précaution: aspects de droit international et communautaire, Paris, 2002

Commission des Communautés europénnes, Communication de la Commission sur le recours au principe de précaution, $\mathrm{COM}(2000) 1,2001$

Coppens, Yves, Rapport de la commission Coppens de préparation de la Charte de l' environnement, Paris, avril 2003

Dupuy, Pierre-Marie, «Le principe de précaution: règle émergente du droit international général », in Leben, Charles/Verhoeven, Joe (dir.), Le principe de précaution : aspects de droit international et communautaire, Paris 2002, p. 95ss

Dutheil de la Rochère, Jacqueline, «Le principe de précaution dans la jurisprudence communautaire », in Leben, Charles/Verhoeven, Joe (dir.), Le principe de précaution : aspects de droit international et communautaire, Paris 2002, p. 193ss

Epiney, Astrid/ Scheyli, Martin, Strukturprinzipien des Umweltvölkerrechts, Baden-Baden, 1998

Fornel (de), Michel/ Passeron, Jean-Claude, L'argumentation : preuve et persuasion, Paris, 2002

Griffel, Alain, Die Grundprinzipien des schweizerischen Umweltrechts, Zurich, 2001

Jonas, Hans, Le principe responsabilité, Paris, 1990

Kerchove (van de), M., «La vérité judiciaire: quelle vérité, rien que la vérité, toute la vérité?», Déviance et société, 2000, no 1, p. 95ss

Kourilsky, Philippe/ Viney, Geneviève, Le principe de précaution: rapport au Premier Ministre, Paris, 15 octobre 1999

Lagarde, Xavier, Réflexions critiques sur le droit de la preuve, Paris, 1994

$99 \quad$ Voir ci-dessus, pp. 5-10. 
Larrère, Catherine, «Le contexte philosophique du principe de précaution», in Leben, Charles/Verhoeven, Joe (dir), Le principe de précaution: aspects de droit international et communautaire, Paris 2002, p. 15 ss

Latour, Bruno, La fabrique du droit: une ethnographie du Conseil d'Etat, Paris, 2002

Lepage, Corinne, «Une Charte qui fait régresser le droit de l'environnement», Le Monde, édition du 15 avril 2003

Le Roy, Yves/ Schoenenberger, Marie-Bernadette, Introduction générale au droit suisse, Zurich, 2002

Lévy-Bruhl, Henri, La preuve judiciaire : étude de sociologie juridique, Paris, 1964

Monediaire, Gérard, «Expertise et droit de l'environnement», in incertitude juridique, incertitude scientifique, Limoges, 2001, p. 107ss

Moor, Pierre, Droit administratif, $1^{\text {cr }}$ volume, $2^{\circ}$ éd., Berne, 1994

Moor, Pierre, Droit administratif, $2^{\circ}$ volume, $2^{\circ}$ éd., Berne, 2002

Morand, Charles-Albert, Le droit néo-moderne des politiques publiques, Paris, 1999

Ost, François/ van de Kerchove, Michel, «De la pyramide au réseau : pour une théorie dialectique du droit», Bruxelles, 2002

Ost, François, «Au-delà de l'objet et du sujet, un projet pour le milieu », in Ost, François/ Gutwirth, S. (éd.), Quel avenir pour le droit de l' environnement? Bruxelles, 1996

Papaux, Alain, Essai philosophique sur la qualification juridique: de la subsomption à l'abduction, Zurich, 2003

Perelman, Chaïm, Ethique et droit, Bruxelles, 1990

Perelman, Chaïm/ Olbrechts-Tyteca, Lucie, Traité de l'argumentation, 5éd., Bruxelles, 1992

Petitpierre, Anne, «Fondements écologiques de 1'ordre constitutionnel Suisse», in Thürer/Aubert/ Müller, Droit constitutionnel suisse, 2001, §36

Piquerez, Gérard, Manuel de procédure pénale suisse, Zurich, 2001

Popper, Karl, La logique de la découverte scientifique, trad. de l'anglais par Nicole Thyssen-Rutten et Philippe Devaux, Paris, 2002

Prieur, Michel, «L'environnement entre dans la Constitution», Droit de l' environnement, 2003

Rausch, Heribert, Kommentar zum Umweltschutzgesetz ad art. 1er LPE, Zurich, 1988

Recanati, François, Les énoncés performatifs: contributions à la pragmatique, Paris, 1981

Ricoeur, Paul, Histoire et vérité, Paris, 1955

Sadeleer (de), Nicolas, Les principes du pollueur-payeur, de prévention et de précaution : essai sur la genèse et la portée juridique de quelques principes du droit de l'environnement, Bruxelles, 1999

Sève, Bernard, «La peur comme procédé heuristique et comme instrument de persuasion », in Hottois, Gilbert (éd.), Aux fondements d'une éthique contemporaine, Paris, 1993, p. 107ss

Solbach, Markus, Politischer Druck und richterliche Argumentation, Francfort, etc., 2003

Trouwborst, Arie, Evolution et Status of the Precautionary Principle in Internation Law, Londres, 2002 